\title{
THE DIG FOR SHAKESPEARE ACADEMIC ADVISORY BOARD ARCHAEOLOGISTS AND VOLUNTEERS
}

\section{Advisory Board}

Nat Alcock, University of Warwick

Robert Bearman, Shakespeare Birthplace Trust

(Chair) Paul Edmondson, Shakespeare Birthplace Trust

Ian George, English Heritage

Tara Hamling, University of Birmingham

Kate McLuskie, University of Birmingham

Nicholas Molyneux, English Heritage

Jonathan Parkhouse, archaeologist

Tiffany Stern, University of Oxford

John Taplin, independent scholar

René Weis, University of London

Stanley Wells, Shakespeare Birthplace Trust

Michael Wood, independent scholar and television historian

\section{Archaeologists}

William Mitchell (Staffordshire University)

Kevin Colls (Staffordshire University)

Elisabeth Charles

Mark Charles

Shane Kelleher

Samantha Paul

John Halsted

Emma Collins

Paul Collins 
Erica Macey-Bracken

Bob Burrows

\section{Volunteers}

Thank you to all of the following volunteers who worked on Dig for Shakespeare between 2010 and 2015 .

John Adams

Russell Andrews

Shelagh Andrews

Gemma Asbury

Elizabeth Ash

Kirsten Ash

Jess Ashton

Tony Atcheson

Michael Athanson

Anicka Backstrom

Robert Baird

Clive Bardell

Adrian Bates

Evelyn Beal

Kimberly Bellamy

Cyril Bennis

Janet Boneham

Jennie Boneham

Karen Boyles

Alexander Brantingham

Gem Brewer

John Brittain

Heather Britton

Martin Broome

Richard Browne

Lizzie Buck

Sue Buck

Chris Burrows

Ellen Callender

Lucy Callender

Colin Campbell

Joan Campbell

Laura Cannon

Sarah Carrington

Peter Chadwick

Sue Chambers

Ryan Charlton

Peter Cheetham
Gill Chew

Louise Clare

Susan Clark

Michael Clough

Lorraine Coles

Antony Collins

Bernadette Collins

Laura Collins

Malcolm Cook

Bethany Coomber

Cheryl Coomber

Clayre Coopey

Eddie Cousins

Louise Cowdell

Anny Crunelle

Ewa Czumaj

Peter Davis

Linda Digby

Jeanette Dobson

Laura Dobson

Louise Dodd

Allan Duff

Eilidh Duff

Margaret Elsy

Tony Estick

Debbie Ewing

Christine Fell

Claire Forkes

Alistair Galt

Emma Gardner

Heather Garrett

Cameron Grant

Anne Grey

Kerry Grocott

Gido Hakvoort

Janet Hall

John Harris

Jonathan Harvey 
John Hawkins

Dominic Heather

Sharland Hewson

James Hicken

Bill Hicks

Alice Hobbs

Matthew Holmes

David Hope

Sara Horaiz

Roger Howells

Trista Huang

Norman Hughes

Karyn Hughes-Jones

Daniel Hume

Alison Hunt

Sarah Jenkins

Ellie Knaggs

Vivienne Lauder

Stephanie Lawrence

Barbara Lister

Nick Lister

Ken Macdonald

Vanessa MacDonald

William Mannin

Joshua Marsh

Linda Martin

Pat Martin

Jane Mason

John May

Fiona McConville

Heather McKechnie

Katie Medlin

Kay Medlin

Brian Miles

Emily Millward

Mary Mills

Anna Moore

Roger Moore

Helen Morgan

Richard Morris

Julie Moss

Nicola Nash

Sally Naylor

Gerald O'Brien

Nina O'Hare

Kelly O'Keefe

Mike Osborne

Sarah Peacop

Driunie Perera
Jennifer Perry

Elizabeth Prater

Louise Ralph

Gemma Reed

Hazel Reed

Tommy Reid

John Richards

Peter Richards

Julia Robins

Rachel Sampson

David Savage

Andrew Scotson

Laura Simcox

Sophie Slater

Jennifer Smith

Lucinda Smyth

Eleanor Stevens

Jeremy Stevens

Gary Stocker

Colin Such

Rita Sweeney

Mark Tanner

Jan Tasker

Eddy Taylor

Jane Taylor

Joyce Taylor

Ken Taylor

Sophie Taylor-Barthorpe

Michelle Thick

Anne Thomas

Rebecca Tillotson

Jann Tracy

Jenny Tyers

Sue Tyler

Kevin Wainwright

Isabel Walker

Maureen Walker

Christine Walsh

Laura Waters

Robin Weaver

Eleanor Webb

Mark Webb

David Westcott

Ken Wheal

Helen Williams

David Wiltshire

Simone Wing

Patricia Wyspianska 